\title{
Ad Hoc Network Routing Algorithm Based on Energy Balanced Connected Dominating Set
}

\author{
Huang Chengbing ${ }^{1}$ \\ ${ }^{1}$ Computer science department of Aba Teachers College , \\ wenchuan sichuan 623002 China \\ E-mail:24951699@qq.com
}

\begin{abstract}
Connected Dominating Set (CDS) is an important means to alleviate the broadcast storm of wireless sensor network. Compared to non-CDS internal nodes, CDS internal nodes have faster energy consumption rates, therefore, the energy level of CDS internal nodes determines the life circle of the network. However, the current CDS algorithms only focus the scale of CDS, and the dominator node of energy level are not considered. For this, this article puts forward the Energy-balance-based Connected Dominating Set Distributed (ECDSD) algorithm. CDS is built based on Weighted Coverage Cost (WCC), which contains the detected energy of neighboring nodes. The nodes with higher WCC values are selected as dominator nodes. According to simulation results, compared with current algorithms, ECDSD algorithm can reduce CDS scale, improve CDS life circle by $23 \%$, and increases the coverage by approximate $33 \%$.
\end{abstract}

Keywords: Wireless sensor network; Life circle; Connected dominating set; Distributed algorithm; Life circle

\section{Introduction}

A large number of sensor nodes are deployed within the field of interest (FOI), then collect the data inside FOI; these sensor nodes make up the wireless sensor network (WSN). Each sensor node has the sensing and communication ability. Sensor nodes collect and process initial data from the environment, then the processed data are transmitted to the destinations. In order to collect the data inside FOI more thoroughly and leaving no blind area, the FOI coverage must be maximized. Sensor nodes may stop working, i.e becoming invalid, due to interference and energy depletion. Therefore, improving the coverage has become a research focus on WSN [1-4]. When CDS is established from the perspective of CDS scale and the problem of network energy balance is ignored, some nodes with excessive residual energy become the dominator nodes. As the energy is very low, such kind of nodes will become invalid very soon, which affects the coverage and life circle of the network. For this, this article puts forward the distributed algorithm ECDSD for establishing the minimum CDS from the perspective of energy balance. In ECDSD algorithm, all nodes at the beginning are in initial state, and when some node works as an initiating node and become the dominating node, sending and receiving information according to its weights, and after determining itself to be a dominator node or dominatee node, it will send different information according to the different states of itself. Such circulation will continue until reaching the convergence of whole network, so that the CDS is formed. The simulation results show that the proposed ECDSD algorithm effectively improves the network coverage and life cycle. 


\section{Constraint Conditions}

It is assumed that homogeneous WSNs have N sensor nodes, which are randomly deployed inside monitoring area A. Each sensor node has an initial energy E, a communication radius $R_{c}$, a sensing radius $R_{s}$, and $R_{c} \geq R_{s}$. All sensor nodes have the same $R_{c}$ and $R_{s}$. The sensing area of sensor nodes covers a circular area with the sensor node as the center and $R_{s}$ as the radius, and the communication area covers a circular area with the sensor node as the center and $R_{c}$ as the radius. In addition, the identifiers used in this article are listed in Table 1.

Definition 1: Coverage: Inside the 2-D plane area A, a sensor node set $S=\left\{s_{1}, s_{2}, \cdots, s_{n}\right\}$ is given. The position coordinate of sensor node $s_{i}(i=1,2, \cdots, n)$ is $\left(x_{i}, y_{i}\right)$. If any point $x$ meets the condition $x \in A\left(s_{i}\right), x$ is covered by $s_{i}$.

Definition 2: Dominating set: A subset $D$ inside undirected graph $G=(V, E)$, and $D \neq \phi$. If $v \in V-D$ and subset $D$ is in the node neighboring to $u$, then $D$ is called a subset of graph $D[11]$.

\section{Table 1. List of Identifiers}

\begin{tabular}{cl}
\hline symbol & description \\
\hline$R_{s}$ & Sensing radius \\
$R_{c}$ & Communication radius \\
$A\left(S_{i}\right)$ & Sensing area of Node $S_{i}$ \\
$N\left(S_{i}\right)$ & 1 step neighboring nodes set of Node $S_{i}$ \\
$C R\left(S_{i}\right)$ & Coverage redundancy set of Node $S_{i}$ \\
$E_{r}\left(S_{i}\right)$ & Residual energy of Node $S_{i}$ \\
$E_{t h}$ & Energy threshold value \\
$E_{t o t}\left(S_{i}\right)$ & Sum of residual energy of neighboring nodes of Node $S_{i}$ \\
$W C C\left(S_{i}\right)$ & Weight value of Node $S_{i}$ \\
$L 1\left(S_{i}\right)$ & Stored 1-step neighboring white node information of Node $S_{i}$ \\
$L 2\left(S_{i}\right)$ & Stored 2-step neighboring white node information of Node $S_{i}$ \\
\hline
\end{tabular}

\section{ECDSD Algorithm}

\subsection{Weights WCC}

Each sensor node periodically broadcasts information packets to its neighboring nodes, and the information packet include residual energy information. In order to reduce packet collision rate, each node randomly set the backoff interval, and after timing, the information packet will be broadcasted. Once receiving the information packet from neighboring nodes, the node will compute its weighted coverage cost (WCC).

$$
E_{\text {tot }}\left(s_{i}\right)=\sum_{\forall x \in C R\left(s_{i}\right)} E_{r}(x), \text { if } E_{r}(x)>E_{t h}
$$

Where, $E_{t o t}\left(s_{i}\right)$ is the residual energy $s_{i}$ in neighboring nodes of node, and $E_{r}(x)$ is larger than the threshold value $E_{t h}$ of all nodes.

Then the WCC of sensor node $s_{i}$ is:

$$
W C C\left(s_{i}\right)=\left\{\begin{array}{lr}
0, & \text { if } E_{r}\left(s_{i}\right)<E_{t h} \\
E_{r}\left(s_{i}\right)+E_{\text {tot }}\left(s_{i}\right), & \text { otherwise }
\end{array}\right.
$$


It can be seen from Formula (2), the WCC value of the node reflects the energy levels of the nodes and neighboring nodes. In order to reduce number of nodes invalid due to energy depletion, set the WCC value of the nodes whose $E_{r}\left(s_{i}\right)$ smaller than threshold value $E_{t h}$ to zero. If residual energy is low, the working time will be short, then it will not need to add CDS set.

As shown in Figure 1, the five sensor nodes, A, B, C, D and E, are distributed inside the network area, and there are coverage and overlapping among them. Assume that their initial energy is all 5J, and Figure 1(a) describes the communication radius (dotted circle) and sensing radius (solid circle) of the node; Figure 1(b) shows the residual energy of each node, for example, the residual energy of Node $\mathrm{C}$ is 2 and the residual energy of Node B is 3. Assuming that $E_{t h}=1$, then the WCC values of each node can be computed according to Formula (2). For example, the sensing are of Node A are overlapped with the sensing areas of Node B, C and D, and the residual energy of these three nodes are all larger than the threshold value, therefore, $W C C(A)=E_{r}(A)+E_{t o t}(A)=4+3+2+1=10$. In the same way, it can be obtained that $W C C(B)=4+3=7, W C C(C)=4+2=6, W C C(D)=4+1=5$ and $W C C(E)=2$.

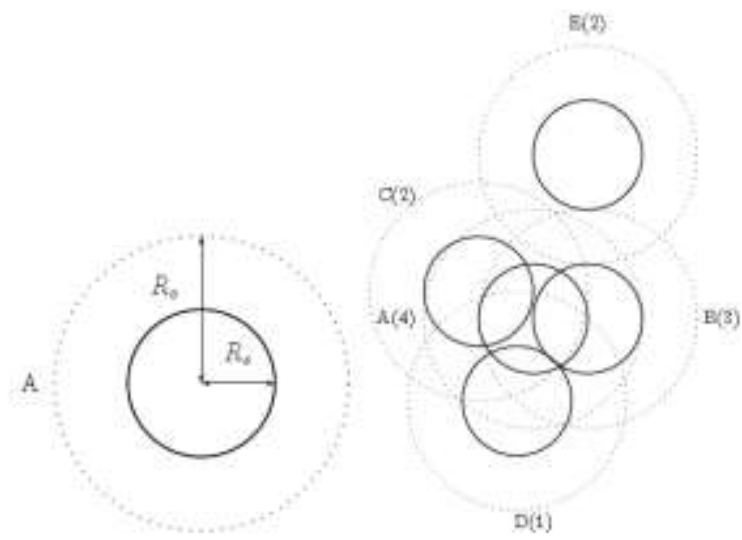

(a) Communication radius and sensing radius (b) Residual energy

Figure 1. Computation Example of WCC Value

\subsection{CDS Building Procedures}

First of all, in order to make clear and vivid description, the nodes in different states are marked by different color, as shown in Table 2 .

Table 2. Status Identification

\begin{tabular}{ccc}
\hline Status & Symbol & Color \\
\hline Initial status & $S_{0}$ & White \\
Dominatee status & $S_{1}$ & Grey \\
Dominator status & $S_{2}$ & Black \\
\hline
\end{tabular}

The core problem of ECDSD algorithm is how to select the next dominator node by using the WCC weights, i.e. the strategy problem for selecting dominator node. In order to make a simple description, it is assumed that Node $i$ is the starting node. At beginning, all nodes are in initial state, and all tags are marked white. Then, Node $i$ finds out the maximum Node $v$ in the information packets received from neighboring nodes, and if the weight value of Node $v$ WCC(v) is larger than the weight value of 
itself $W C C(i)$, Node $v$ will broadcast the packet Mes_Dominator and declares Node $v$ as the dominator node; otherwise Node $i$ will broadcast packet Mes_Dominator and declares itself as the dominator node.

As shown in Figure 2, at first, each node is marked white, and the Node 14 in the graph is the Node $i$, namely $i=14$. In order to make the description concise, the weight of each node is their number ID. As Node $i$ has the largest weight among neighboring nodes, it broadcasts the packet Mes_Dominator and becomes the dominator node, therefore, it is marked in black color.

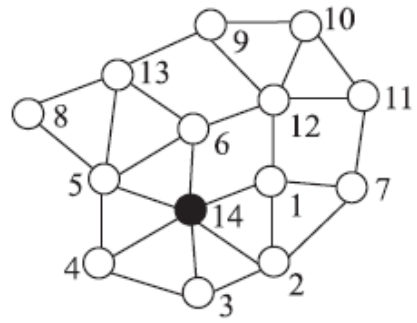

Figure 2. Generation of the First Dominator Node

After generating the first dominator node, this node will broadcast the Mes_Dominator packet. The neighboring nodes which receive this Mes_Dominator packet will temporarily become the dominatee nodes and broadcast the Mes_Dominatee packet, and they will be marked in grey color. Still take Figure 2 as an example, neighboring nodes $1,2,3,4,5$ and 6 become dominatee nodes after receiving the Mes_Dominator packet, and they are marked in grey color, as shown in Figure 3.

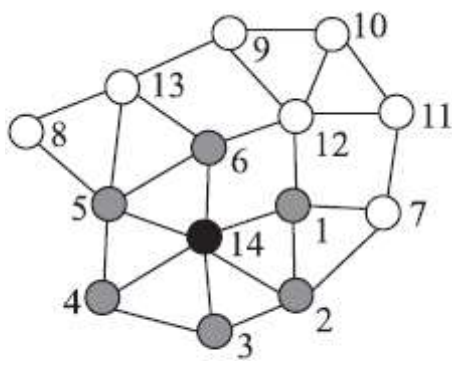

\section{Figure 3. Generation of the Dominatee Node (First Round)}

Once a white node receives the Mes_Dominatee packet, it will broadcast its packet. Take Figure 3 as an example, white nodes 8,13, 12 and 7 will broadcast their packets. Therefore, grey nodes will broadcast receive packets broadcasted from neighboring nodes, and store these packets in List L1, then grey nodes will broadcast List L1. For example, the Node 6 in Figure 3 receives the packets broadcasted from Node 13 and 12, then it stores their weights and ID numbers in its List L1. Then it will broadcast the List L1.

Then, the first dominator node, namely the black node will receive the List L1 broadcasted by each grey nodes, and store List L1 in List L2. The black node will find out the white node with the largest weight in List L2, and this white node will be selected as the second dominator node and marked black. For example, Node 13 in Figure 3 is the white node with the biggest weight in List L2, therefore, it becomes the second dominator node.

Finally, the grey node, which is among the one step neighboring nodes ${ }^{N(14)}$ from the first dominator node (14) and is neighboring to the second dominator node ${ }^{(13)}$ and 
has the biggest weight, becomes the third dominator node. It can be seen from Figure 3 that Node 5 and 6 are neighboring nodes to Node 14 and 13, and Node 6 has a higher weight than Node 5, therefore, Node 6 becomes the third Node, as shown in Figure 4. Repeat the above steps until no white nodes existing in the network. Then all black nodes make up the CDS set. Still take Figure 3 as the example, the final dominator nodes are as shown in Figure 4, and Node 12 and 11 become the fourth and fifth dominator node, respectively.

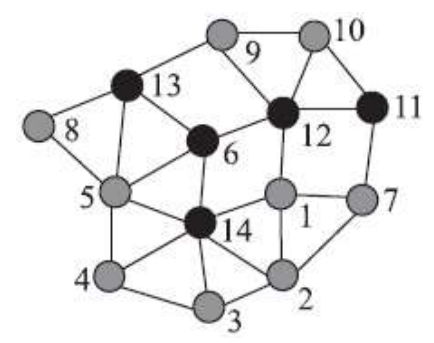

Figure 4. Generation of Dominator Nodes

The above steps for building CDS set are as shown in Figure 5.

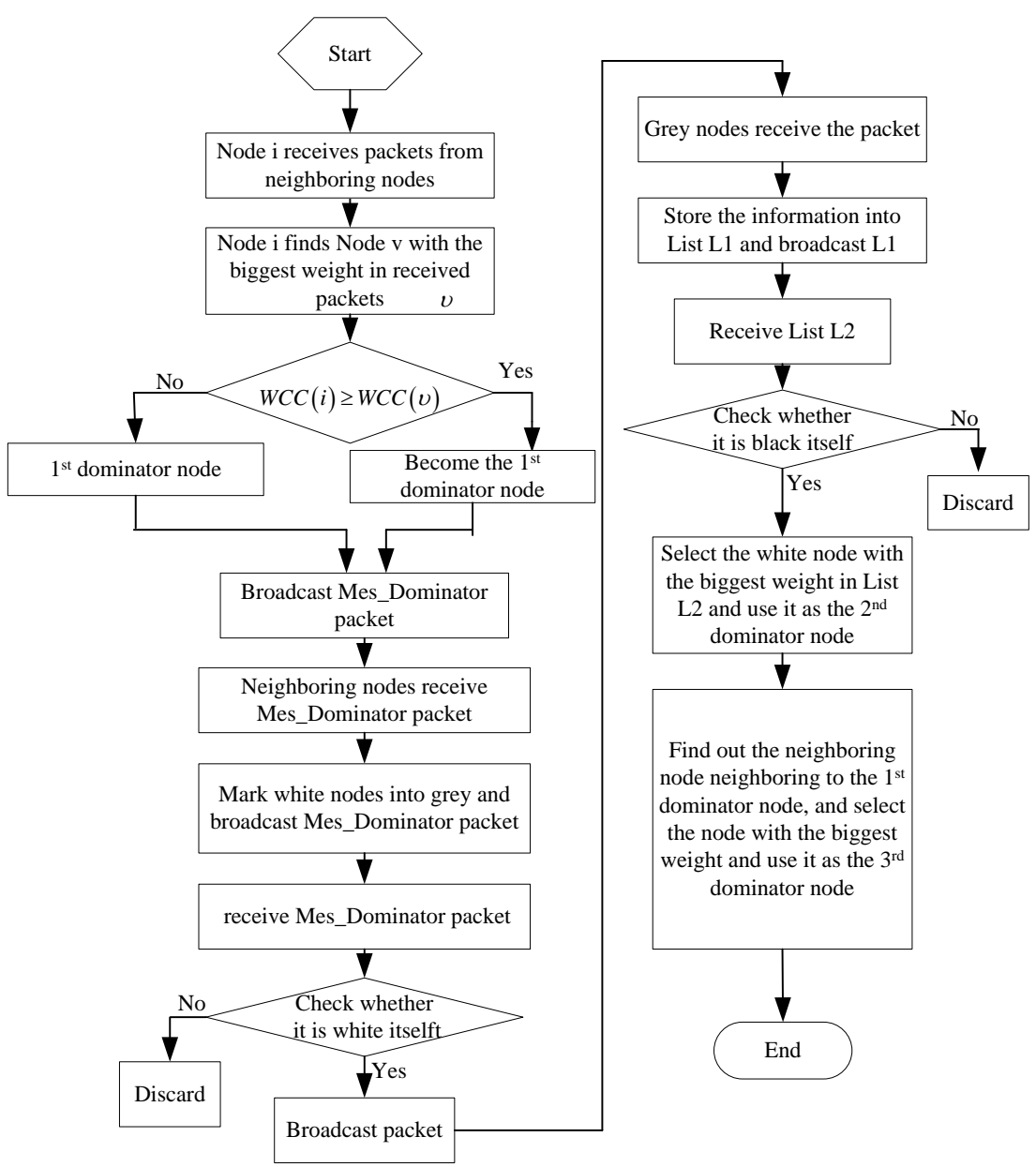

Figure 5. Flow Chart for Generating Three Dominator Nodes 


\section{Performance Analysis}

\subsection{Simulation Parameters}

The simulation on ECDSD algorithm is conducted in this Chapter. Assume that $\mathrm{N}$ sensor nodes are randomly deployed in a 2D plane, and region area is $A=200 \times 200 \mathrm{~m}^{2}$. Simulation parameters are shown in Table 3. Each independent simulation is repeated for100 times, and then the mean value is used as final experimental data.

Table 3. Simulation Parameters

\begin{tabular}{cc}
\hline Parameter & Value \\
\hline Simulation area & $200 \mathrm{~m} \times 200 \mathrm{~m}$ \\
Node distribution & random \\
Number of nodes & $100 \sim 400$ \\
Communication range & $40 \sim 70$ \\
Residual energy of the node & $1 \mathrm{~J}$ \\
Data packet size & 100 byte \\
Control packet size & 25 byte \\
\hline
\end{tabular}

In the process of performance analysis, mean CDS scale, CDS life circle, residual energy, region coverage and CDS convergence time are used as the performance index. Both EB-CDS [9] and HMCDS [10] algorithms are used as reference.

\subsection{Data Value Analysis}

Figure 6 shows the mean CDS scale of three algorithms, and it can be seen from Figure 9 that, ECDSD algorithm are slightly better than EB-CDS and HMCDS algorithms as ECDSD algorithm can exclude the nodes whose energy are lower than threshold value from CDS set, which reduces the number of active nodes.

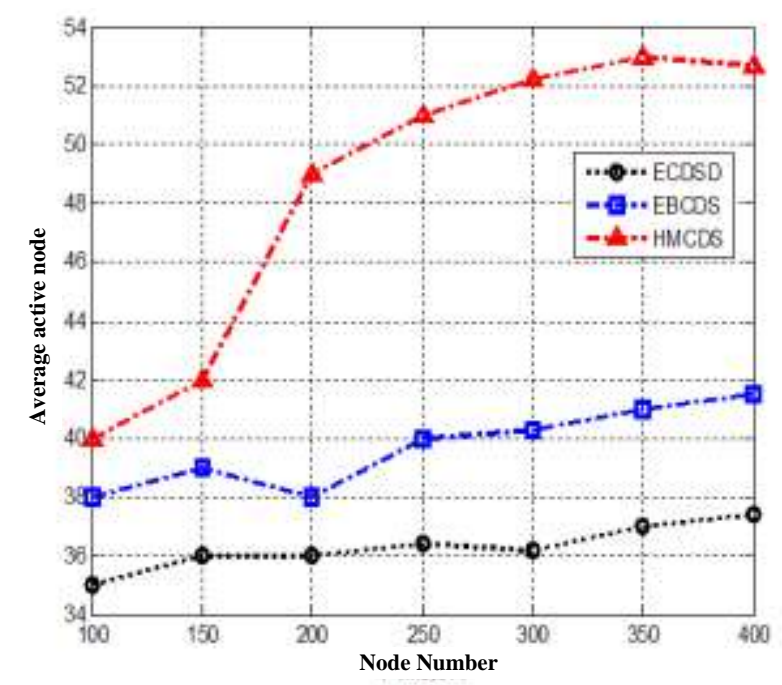

Figure 6. CDS Scale

Figure 6 shows the CDS convergence time. It can be seen from Figure 7 that, with the increasing of nodes, the convergence time of three algorithms are also increasing, 
and their convergence time are similar. But when the number of nodes exceeds 300 , ECDSD algorithm obviously shows a better rapid convergence performance. It is because ECDSD algorithm adopts simpler rules in building CDS set, which means high efficiency.

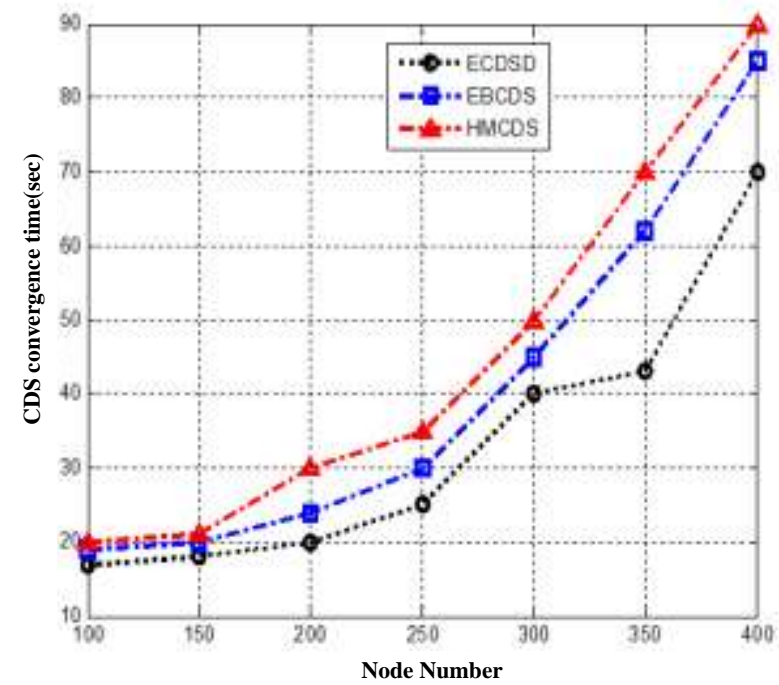

Figure 7. CDS Convergence Time

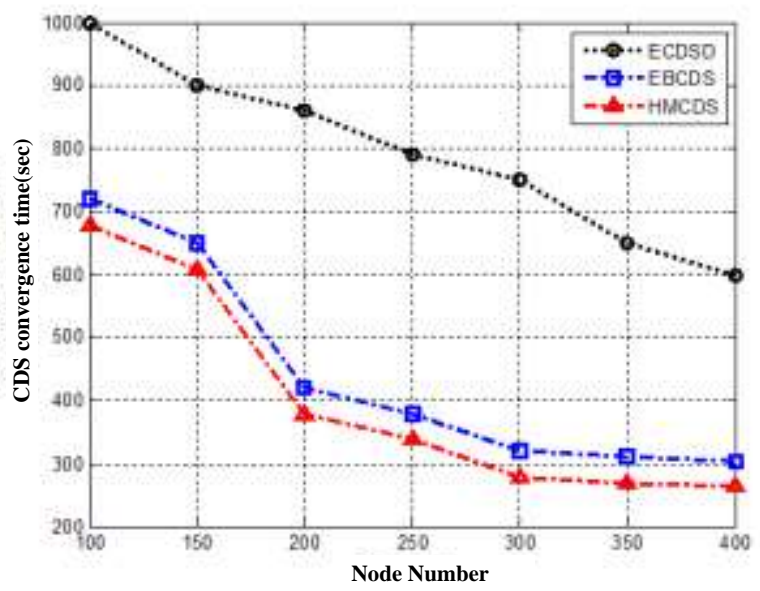

Figure 8. CDS Life Cycle

Figure 8 shows the curve of CDS life circles of three algorithms vary with the change of the number of nodes. It can be seen from Figure 8 that, with the increasing of the number of nodes, corresponding number of active nodes also increases, and more energy are consumed and CDS life circle declines. And compared with EB-CDS and HMCDS algorithms, ECDSD algorithm has longer life circle as it uses weight value to select the dominator nodes, which means that only the nodes with large coverage weight values can become active nodes. 


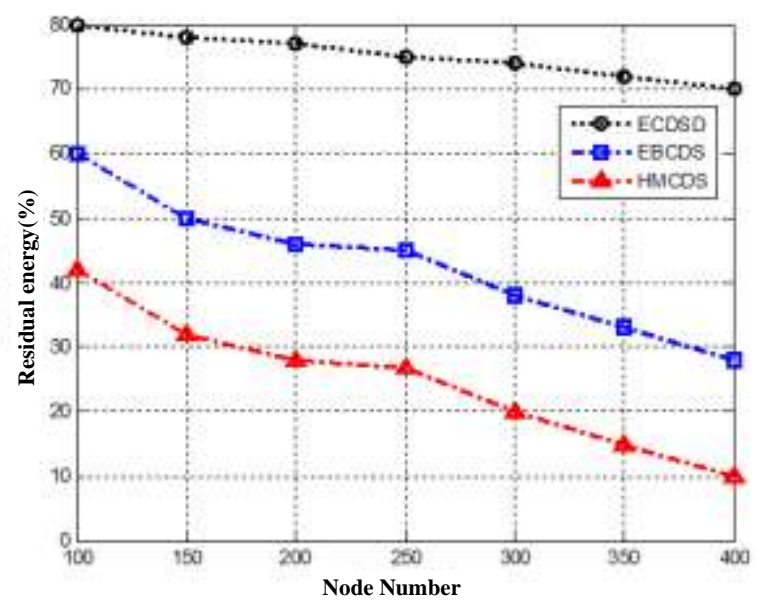

Figure 9. Residual Energy

Figure 9 shows the residual energy of the network after carrying out the simulation for 7200 seconds. It can be seen form Figure 9 that ECDSD algorithm has the highest residual energy. That is because ECDSD algorithm alleviates the load of active nodes, saving energy consumption of the nodes.

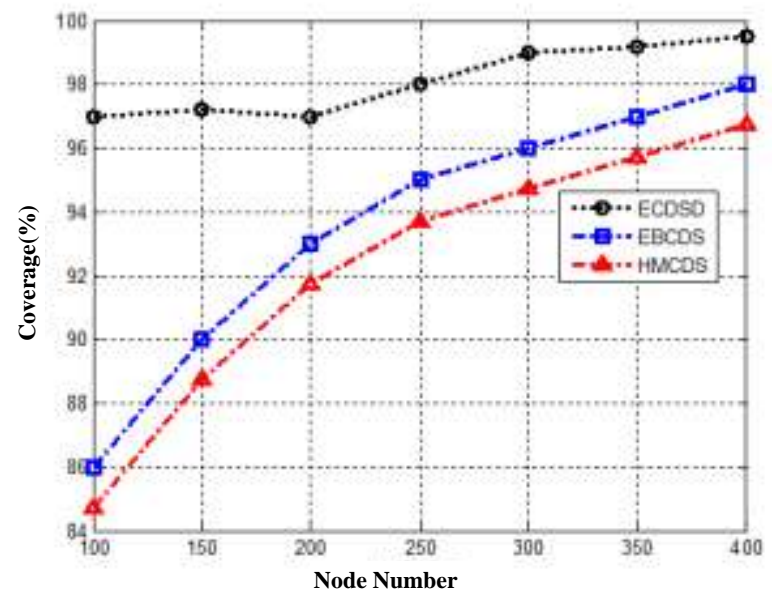

Figure 10. Coverage

At last, Figure 10 shows the curve of region coverage varying with the change of the number of nodes. With the increase of number of nodes, coverage also increases. Compared with HMCDS and EBCDS algorithms, ECDSD algorithm requires less active nodes, and accordingly, it consumes less energy in the same time period, and ECDSD algorithm selects the nodes with high coverage weights as the active nodes. It can be seen from Figure 10 that when there are only a few nodes, three algorithms all have low coverage, and with the increasing of the number of nodes, the coverage rates begin to rise. ECDSD algorithm keeps a region coverage of $97 \%$, which is better than those of HMCDS and EBCDS algorithms.

\section{Conclusion}

This article puts forward the Energy-balance-based Connected Dominating Set Distributed (ECDSD) algorithm, which considers the energy distribution of nodes. It tries to include the nodes whose residual energy are higher than threshold value in the 
dominator set. ECDSD algorithm uses the WCC to select the dominator nodes, and as WCC contains the energy information of neighboring nodes, it can balance the energy consumption of the network. The simulation results show that the proposed ECDSD algorithm effectively improves the network coverage and extends the life cycle of the network.

\section{Acknowledgement}

Project supported by the Education Department of Sichuan Province Natural Science Fund (14ZA0306); Aba Teachers College Focus Fund (ASA12-23)

\section{References}

[1] D. Jiang, X. Ying and Y. Han, "Collaborative multi-hop routing in cognitive wireless networks", Wireless Personal Communications, (2015), pp. 1-23.

[2] J. Hu and Z. Gao, "Modules identification in gene positive networks of hepatocellular carcinoma using Pearson agglomerative method and Pearson cohesion coupling modularity", Journal of Applied Mathematics, (2012).

[3] D. Jiang, Z. Xu and Z. Chen, "Joint time-frequency sparse estimation of large-scale network traffic", Computer Networks, vol. 55, no. 15, (2011), pp. 3533-3547.

[4] J. Hu, Z. Gao and W. Pan, "Multiangle Social Network Recommendation Algorithms and Similarity Network Evaluation", Journal of Applied Mathematics, (2013).

[5] M. Zhou, G. Bao, Y. Geng, B. Alkandari and X. Li, "Polyp detection and radius measurement in small intestine using video capsule endoscopy", 2014 7th International Conference on Biomedical Engineering and Informatics (BMEI), (2014).

[6] G. Yan, Y. Lv, Q. Wang and Y. Geng, "Routing algorithm based on delay rate in wireless cognitive radio network", Journal of Networks, vol. 9, no. 4, (2014), pp. 948-955.

[7] Y. Lin, J. Yang and Z. Lv, "A Self-Assessment Stereo Capture Model Applicable to the Internet of Things", Sensors, vol. 15, no. 8, (2015), pp. 20925-20944.

[8] K. Wang, X. Zhou, T. Li, "Optimizing load balancing and data-locality with data-aware scheduling", Big Data (Big Data), 2014 IEEE International Conference on. IEEE, (2014), pp. 119-128.

[9] L. Zhang, B. He and J. Sun, "Double Image Multi-Encryption Algorithm Based on Fractional Chaotic Time Series", Journal of Computational and Theoretical Nanoscience, vol. 12, (2015), pp. 1-7.

[10] T. Su, Z. Lv and S. Gao, "3d seabed: 3d modeling and visualization platform for the seabed", Multimedia and Expo Workshops (ICMEW), 2014 IEEE International Conference on. IEEE, (2014), pp. 1-6.

[11] Y. Geng, J. Chen, R. Fu, G. Bao and K. Pahlavan, "Enlighten wearable physiological monitoring systems: On-body rf characteristics based human motion classification using a support vector machine", IEEE transactions on mobile computing, vol. 1, no. 1, (2015), pp. 1-15.

[12] Z. Lv, A. Halawani and S. Feng, "Multimodal hand and foot gesture interaction for handheld devices", ACM Transactions on Multimedia Computing, Communications, and Applications (TOMM), vol. 11, no. 1s, (2014), p. 10.

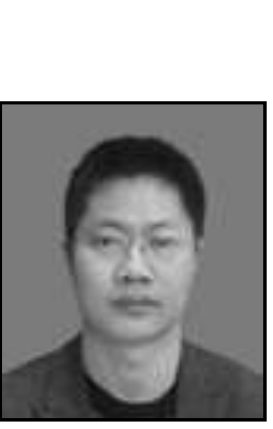

\section{Author}

Huang ChengBing, he is a master of Southwest Jiao Tong University, associate professor of computer science, the senior member of CCF.

Engaged in the research of computer network technology. Presided over the research projects of provincial level and provincial teaching reform project of each 1; university key scientific research project 2, 1 teaching reform project; participation all provincial university research topic 6 items; editor in chief of national higher vocational education computer programming teaching materials 1 . In various journals published nearly 20 . 
International Journal of Future Generation Communication and Networking Vol. 10, No. 1 (2017) 